\title{
First cosmogenic neutrino limits from the ARA Testbed station at South Pole
}

\author{
David Besson, Amy Connolly, Jonathan Davies, Eugene Hong, Carl Pfendner* for \\ the ARA Collaboration \\ The Ohio State University \\ E-mail: pfendner.1@osu.edu
}

\begin{abstract}
The Askaryan Radio Array (ARA) is an ultra-high energy (UHE) cosmic neutrino detector located at the South Pole. The cosmic ray flux cut off above primary energies of $10^{19.5} \mathrm{eV}$ leads us to expect a UHE neutrino flux due to the Greisen-Zatsepin-Kuzmin (GZK) effect. The detection of these UHE cosmic neutrinos will add to the understanding of the sources and physics of UHE cosmic rays. The radio Cherenkov technique is the most promising technique for a long term program to investigate the UHE cosmic neutrino flux. ARA uses this radio Cherenkov technique by deploying radio frequency antennas at a depth of $200 \mathrm{~m}$ in the Antarctic ice. A prototype ARA TestBed station was deployed in the 2010-2011 season and the first three ARA stations were deployed in the 2011-2012 and 2012-2013 seasons. We present the results of the first neutrino search with ARA, using data taken from 2011-2012 with the ARA TestBed station.
\end{abstract}

The 34th International Cosmic Ray Conference,

30 July- 6 August, 2015

The Hague, The Netherlands

${ }^{*}$ Speaker. 


\section{Introduction}

The Askaryan Radio Array (ARA) aims to measure the flux of ultra-high energy (UHE) neutrinos above $10^{17} \mathrm{eV}$. While UHE neutrinos are so far undetected, they are expected both directly from astrophysical sources and as decay products from the GZK process [1]. , as first pointed out by Berezinsky and Zatsepin [2]. The GZK process describes the interactions between cosmic rays and cosmic microwave and infrared background photons above $\mathrm{a} \sim 10^{19.5} \mathrm{eV}$ threshold.

The interaction of a UHE neutrino in dense media induces an electromagnetic shower which in turn creates impulsive radio-frequency (RF) Cherenkov emission via the Askaryan effect [3]. In radio transparent media, these RF signals can then be observed by antenna arrays read out with $\sim \mathrm{GHz}$ sampling rates.

Currently, the most stringent limits on the neutrino flux above $10^{19} \mathrm{eV}$ have been placed by the balloon-borne ANITA experiment sensitive to impulsive radio signals from the Antarctic ice sheet [4]. Below $10^{19} \mathrm{eV}$, the best constraints on the neutrino flux currently come from the IceCube experiment. IceCube has recently reported the first cosmic diffuse neutrino flux, which extends up to $\sim 10^{15} \mathrm{eV}[5]$.

Due to the $\sim 1 \mathrm{~km}$ radio attenuation lengths in ice [6,7], radio arrays have the potential to view the 100 s of $\mathrm{km}^{3}$ of ice necessary to reach the sensitivity to detect $\sim 10$ events per year from expected UHE neutrino fluxes. Next-generation detectors are under construction aiming to reach the 100s of $\mathrm{km}^{3}$ target volume of ice. The Askaryan Radio Array (ARA) [6] is one such detector being deployed in the ice at the South Pole and the first physics results from a prototype station of this detector are presented in these proceedings.

ARA aims to deploy 37 stations of antennas at $200 \mathrm{~m}$ depth spanning $100 \mathrm{~km}^{2}$ of ice as shown in Fig. 1. A design station consists of eight horizontally polarized (HPol) and eight vertically polarized (VPol) antennas at depth as shown in Fig. 2.

To date, one ARA prototype Testbed station and three full stations have been deployed in the ice. The Testbed station was deployed at a depth of $\sim 30 \mathrm{~m}$ in the 20102011 drilling season. The first full station, A1, was deployed at a depth of $100 \mathrm{~m}$ in the 2011-2012 drilling season. The next two stations, A2 and A3, were deployed at the $200 \mathrm{~m}$ design depth during the 2012-2013 season.

\section{Testbed}

The ARA prototype Testbed station differs from the layout of the design stations for 
the full array (see Fig. 3). As with the deep

stations, the Testbed antennas deployed in boreholes were designed to be broadband, with a mixture of HPol and VPol. The VPol antennas are a wire-frame hollow-center biconical design with a bandwidth of 150-850 MHz. Four were deployed in boreholes and 2 at the surface. For HPol, two designs were used in the Testbed, the bowtie-slotted cylinder (BSC) and the quad-slotted-cylinder (QSC). The BSCs were used in four borehole antennas and a pair of QSC's in the fifth borehole. A more complete description of the design and operation of the Testbed station can be found in [6].

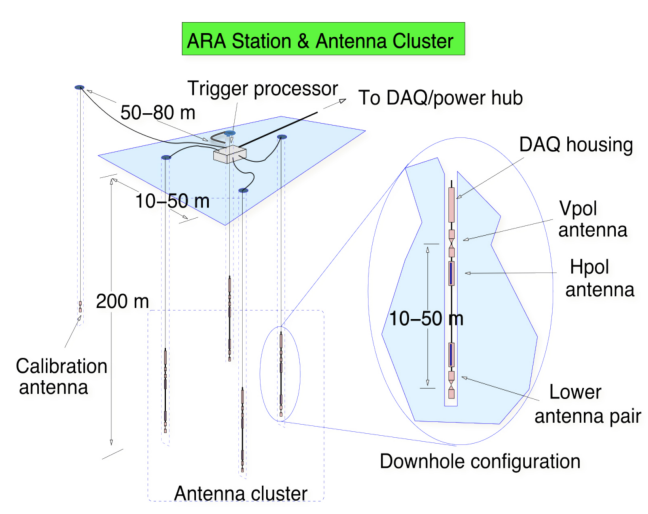

Figure 2: Diagram showing the layout of a single ARA station.

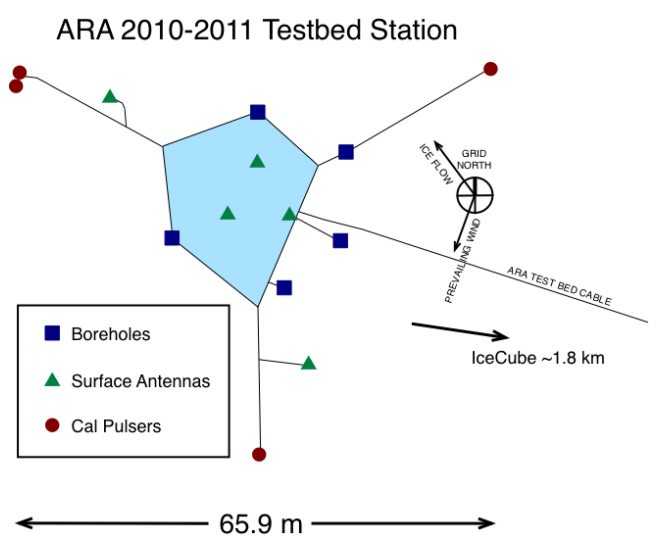

Figure 3: Schematic of the ARA Testbed station.

Three calibration pulser VPol and HPol antenna pairs were installed at a distance of $\sim 30 \mathrm{~m}$ from the center of the Testbed array to provide in situ timing calibration and other valuable cross checks related to simulations and analysis. The pulser produces a $\sim 250 \mathrm{ps}$ broadband impulsive signal at a rate of $1 \mathrm{~Hz}$ that can be observed by the receiver antennas.

The entire signal chain from is modeled using a simulation package known as AraSim. This software models the RF emission from the particle shower, propagates the signal through the ice, and models the antenna and electronics response to the received signal.

\section{Testbed Data Analyses}

Three analyses of 2011-2012 Testbed were performed. The first analysis (the Interferometric Map Analysis) assesses the quality of reconstructions from interferometric maps based on depthdependent ray tracing and we use the results of this analysis to derive constraints on the neutrino flux at the conclusion of the paper. The Coherently Summed Waveform Analysis uses a different reconstruct technique, performing a best fit to time delays derived from coherently summed waveforms. These two analyses examine data from January 2011 to December 2012 The third analysis, the Template-Based Analysis, performs correlations between events and searches for any producing a unique pattern of waveforms. This analysis only examines data from March to August 2011. A complete description of these analyses can be found in [8].

\subsection{Interferometric Map Analysis with Depth Dependent Ray Tracing}

The first of the three analyses reconstructs events using an interferometric map technique. For 
this analysis, we consider RF triggered events from January $8^{\text {th }}$, 2011 to December $31^{\text {st }}, 2012$ and use a set of optimized cuts using AraSim calibrated against Testbed data to eliminate background events from our final sample. The total analysis livetime for this period is 415 days. This analysis is performed in two stages. Stage 1 was a complete analysis on a limited data set that had been processed at an early period of data processing. A complete analysis is carried on data from from February-June of 2012 only, optimizing cuts on the $10 \%$ set before opening the box on that time period alone. In Stage 2, the cuts were re-optimized on the $10 \%$ set for the two year period but excluding February-June 2012 which had already been analyzed.

In order to reconstruct the interaction

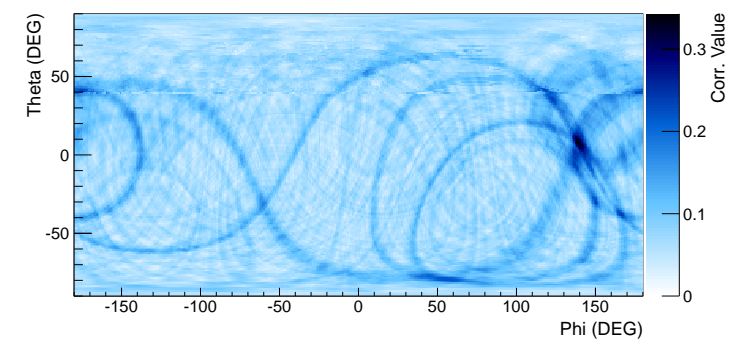

Figure 4: An example of a interferometric map used for reconstruction. This event is a calibration pulser and reconstructs well to the appropriate location in this $30 \mathrm{~m}$ inteferometric map where the correlation value is high (dark blue). location of the event, we use the crosscorrelation of the two antennas to characterize the signal delay between them. For each event, the cross-correlations are then summed for all pairs of the same polarization and are mapped to the antenna delays from putative source distances of $3 \mathrm{~km}$ and $30 \mathrm{~m}$ only (see Fig. 4). We use the $30 \mathrm{~m}$ map to reject improperly flagged calibration pulser signals, and the $3 \mathrm{~km}$ map to determine the reconstruction direction of distant sources, such as neutrinos. For each $1^{\circ} \times 1^{\circ}$ bin in the map, we sample the correlation function for each pair of antennas at the delay expected for that source direction and distance, and form the summed cross-correlation that is entered on the map. We define the reconstruction direction to be the location of the peak in the correlation map. Based on the calibration pulser events, our pointing resolution on the RF source direction is $\sim 1^{\circ}$.

We use the features of the created maps to characterize the quality of the reconstruction and require that the signal direction dominates the reconstruction map. The Reconstruction Quality Cuts require that the area surrounding the peak where the correlation remains above $85 \%$ of the peak, $A_{\text {peak }}\left(\right.$ degrees ${ }^{2}$ ) be greater than $1 \mathrm{deg}^{2}$ and less than $50 \mathrm{deg}^{2}$. Furthermore, we define the total area on the map showing high correlations above that same $85 \%, \mathrm{~A}_{\text {total }}$. The second condition for the Reconstruction Quality Cut requires the ratio between $\mathrm{A}_{\text {total }}$ and $\mathrm{A}_{\text {peak }}$ to be less than 1.5.

Additional cuts are included to decrease identified background signals. A set of Geometric Cuts reject events that reconstruct to locations where background due to anthropogenic noise is expected to be high, either where there is known human activity (e.g. South Pole Station) or where signals reconstruct to the same location repeatedly without an identifiable source. Three such repeating locations were identified and geometric cuts were designed to reject events from these locations. Descriptions of further cuts can be found in [8]. After a number of quality cuts, the effective livetime drops to 224 days.

As a last cut, a Peak/Correlation Cut is applied. Since we expect impulsive events to exhibit a correlation between the $\mathrm{V}_{\text {peak }} / \mathrm{RMS}$ values from the waveforms and maximum correlation value from the reconstruction map, we designed a cut using these two values, as in [4]. 

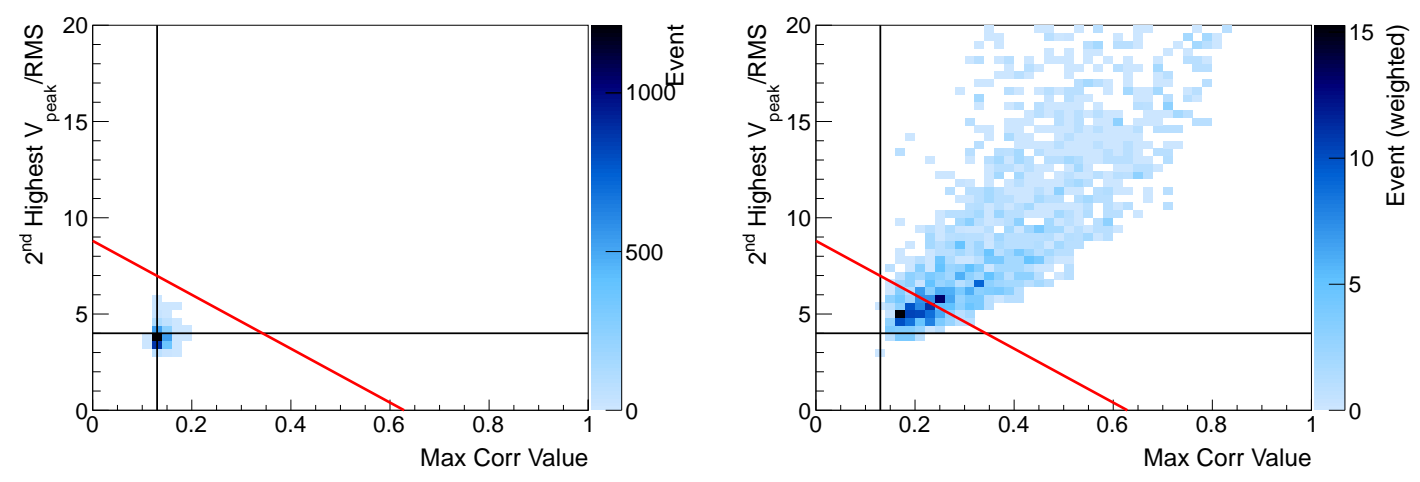

Figure 5: The distribution of 2nd highest $V_{\text {peak }} / \mathrm{RMS}$ and correlation values for the vertical polarization channel for (a) the $10 \%$ examination data set and (b) events simulated at $10^{18} \mathrm{eV}$. Both plots show only events that have survived all other cuts. The red line shows the selected cut parameter and thus all events above this line survive the cuts and those below are removed. For the data (a), no events fall above the cut line. For the simulated events (b), there is a sizable percentage of events that lie above the cut line and thus survive the analysis.

The Peak/Correlation Cut is based on a 2-dimensional scatter plot that has $2^{\text {nd }}$ highest $\mathrm{V}_{\text {peak }} / \mathrm{RMS}$ on the vertical axis and a maximum correlation value on the horizontal axis for the corresponding polarization (see Fig. 5). First, we set constant cuts at $2^{\text {nd }}$ highest $V_{\text {peak }} / R M S>4.0$ and maximum correlation value $>0.13$. We use the $2^{\text {nd }}$ highest $V_{\text {peak }} / R M S$ instead of the highest so that two channels exceed our threshold. After this, we define a cut as a line on the plot of $\mathrm{V}_{\text {peak }} / \mathrm{RMS}$ vs. maximum correlation as shown in the figures. Events located above this line will pass the cut.

We choose the Peak/Correlation Cut that gives us the best expected limit on the maximal model from Kotera et al. 2010 [9]. The optimal vertical offset gives us 0.06 estimated background events and 0.02 expected neutrino events from the Kotera maximal model in the $90 \%$ data set in the Stage 2 analysis.
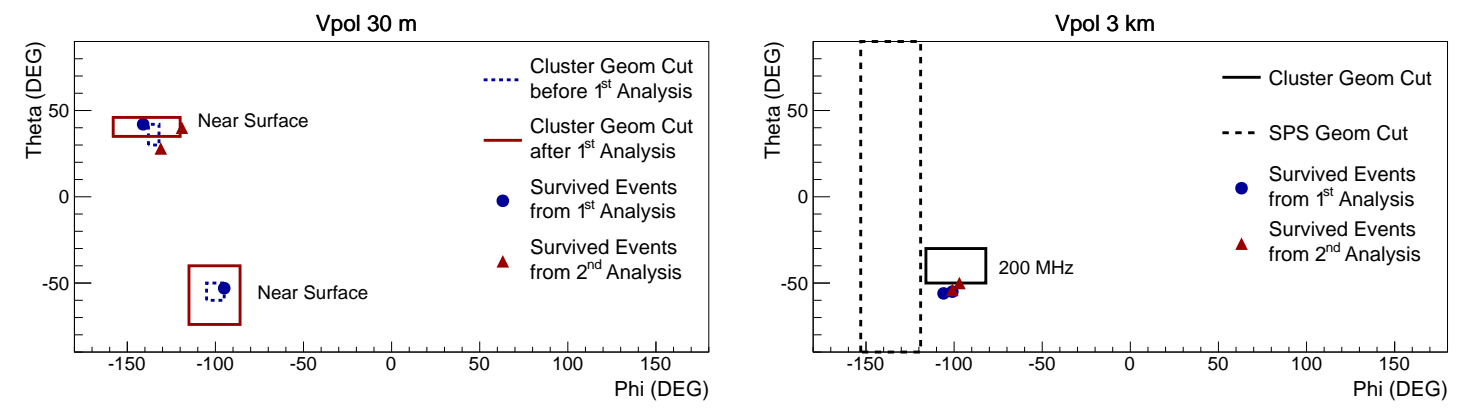

Figure 6: The reconstruction directions of the events that passed both Stage 1 and Stage 2 of the analysis in the $30 \mathrm{~m}$ (upper) and $3 \mathrm{~km}$ (lower) maps. Events that passed the unaltered cuts in Stage 1 are shown in blue and those that passed the Stage 2 cuts are shown in red. The initial Geometric Cut regions (dashed blue line) were adjusted after Stage 1 (solid red lines) based on a Gaussian fit to the background event distribution with a limited set of cuts applied.

In Stage 1 of the analysis, we had three events survive all cuts and all were recognizable back- 
ground signals. These three events were all known types of anthropogenic impulsive events, and one was removed by adjusting the Gradient Cut and the other two by altering the Geometric Cut. In Stage 2 of the analysis (2011-2012), using these new Geometric Cut regions, two events survived, which again were recognizable background signals. The four events that were rejected by the modified Geometric Cuts in the first and second stages can be seen in Fig. 6, along with the Geometric Cut regions. The alterations to the Geometric Cut regions increase the total acceptance of the Geometric Cut (which includes the South Pole region) by less than 5\%. After these modifications, zero neutrino candidate events survived. In future analyses, we plan to design cuts to reject these type of events by other means, with less reliance on the Geometric Cuts.

\subsection{Coherently Summed Waveform Analysis}

The Coherently Summed Wave Analysis differs from the Interferometric Map Analysis in its reconstruction method, continuous wave rejection and other cut parameters. The initial data quality cuts and trigger timing cuts (to reject calibration signals) are performed in a similar manner and will not be discussed here.

In order to remove $\mathrm{CW}$ contaminated events, a probability is calculated on an event by event basis that the measured frequency content is thermal in nature. Events are then rejected when an excess is observed across a narrow range of frequencies. The probability threshold and minimum width are tuned using a combination of events identified as containing a known $\mathrm{CW}$ source, calibration pulser events and simulated neutrino signals to avoid rejecting broadband signals.

The reconstruction method is based upon calculating timing offsets between waveforms that maximize correlation. This is achieved by creating a coherently summed wave (CSW), where individual antenna waveforms are added, offset in time relative from one another. These offsets are computed using a simple algorithm that finds a CSW that is maximally correlated with the individual antenna waveforms, as measured by cross-correlations. The timing difference between pairs of antennas holds information about the arrival direction of the radio signal and are checked against those calculated from an ice model using uniform index of refraction. A $\chi^{2}$ is computed for a series of trial source locations in 1 degree bins in $\theta, \phi$ and logarithmically spaced bins in radial distance $R$. The reconstructed location is that which minimizes the $\chi^{2}$ and hence corresponds to the most likely physical location given the measured time offsets. Thermal events will have essentially random offsets and thus the reconstruction location will have a large $\chi^{2}$. Thus low $\chi^{2}$ values indicate reconstruction consistent with a definite source location.

A CSW is formed for the HPol and VPol antennas separately and two parameters are derived that are used to identify neutrino-like signals. The first parameter is the peak voltage in the CSW, which acts as a measure of power in the constituent antennas. The cross-correlation waveform is computed for each antenna with the CSW of the remaining antennas. The maximum cross-correlation is found in each of these waveforms and summed which acts as a measure of coherence. A linear combination of these parameters, dubbed 'Powherence', is taken to maximize the separation between thermal events and a combination of simulated neutrino and calibration pulser events.

Having applied the CW, $\chi^{2}$ and Powherence Cuts to the VPol and HPol antennas separately, cuts are made to remove time periods and directions producing large numbers of passing events. The CSW reconstruction achieves $\sim 1$ degree resolution for both simulated neutrino events and 
calibration pulser signals. A 50 degree region in azimuth corresponding to the direction of the IceCube Laboratory, as well as 10 degree regions around calibration pulser locations were masked off. In addition, events are rejected where the reconstructed source location is above the ice.

One event survives the final cuts in the analysis, but upon inspection it is clearly due to an anthropogenic $\mathrm{CW}$ signal that narrowly passes the dedicated $\mathrm{CW}$ Cut due to the presence of two carrier frequencies, hence is rejected and thus is not a neutrino candidate event. The total effective livetime for this analysis was 196 days.

\subsection{Template-Based Analysis}

The third analysis strategy presented in this paper traces its heritage to the RICE experiment, which defined 'background' generically as any repetitive waveform or hit antenna pattern. In this approach, a sequence of event-selection criteria are initially applied to suppress both anthropogenic and thermal noise relative to 'interesting' events (either in-ice neutrino interactions, typically coming from below a given ARA station, or perhaps down-coming radio signals from extensive air showers (EAS)) as follows.

First, $\mathrm{CW}$ contamination is reduced by filtering any $\mathrm{CW}$ line which has more than $8 \%$ of the total power in the frequency spectrum, and then continuing with the analysis on that filtered event. Next, triggered events must have at least four antennas with voltage excursions larger than $6 \times$ the root-mean-square voltage $\sigma_{V}$. The $\sigma_{V}$ for a particular antenna is measured using forced triggers (and excluding CW contributions).

Second, triggered events must have a well-reconstructed, single source vertex point, as defined by the event $\chi^{2}$ (defined below), and using source identification algorithms based on RICE codes. In this source reconstruction scheme, antennas are assigned a "hit-time" corresponding to the time at which the voltage magnitude exceeds $6 \sigma_{V}$. The source vertex point $\mathbf{r}_{\mathbf{S}}$ for an event occurring at time $t_{S}$ is determined in three complementary ways:

1. Using the CERN-based MINUIT minimization package, we find the space point which minimizes the sum of the propagation-time residuals, assuming that vertex point, i.e. we minimize $\chi^{2}=\sum_{i}\left(t_{S}-\left[t_{i}-\left|\mathbf{r}_{\mathbf{S}}-\mathbf{r}_{\mathbf{i}}\right| / c\right]\right)^{2}$, where $t_{S}$ is the calculated propagation time from the putative source point to the $i^{\text {th }}$ antenna, $t_{i}$ is the measured time for that antenna as defined by the first $6 \sigma_{V}$ criterion outlined above, $\mathbf{r}_{\mathbf{S}}$ is the putative source point in coordinate space, $\mathbf{r}_{\mathbf{i}}$ is the known location for the $i^{\text {th }}$ antenna, and the sum runs over all the hit antennas.

2. Second, we find that space point defined as the centroid of the event vertices defined by subsets of four hits of the same polarization. That space point can be thought of as the intersection point of spheres centered on each hit antenna, with a spherical radius $r=c(t-$ $\left.t_{0}\right)$, and $t_{0}$ the time of the in-ice neutrino interaction.

3. The results of the previous two calculations are compared against the reconstructed source location using standard ARA interferometric techniques.

The third event-selection criterion requires a total minimum waveform power (defined as $\Sigma\left(V_{i}^{2}\right)$ for all the in-ice antennas) to suppress any thermal noise events which pass the four-fold $6 \sigma_{V}$ requirement. Next, if the source location for events passing the previous two requirements 
is consistent with the known location of the englacial calibration pulser, the event is rejected as a pulser event.

In the final step, triggered events passing the first four requirements are then compared to all other events satisfying those requirements. If the two events are 'similar' (as defined by a direct dot product between the two event waveforms, or by the timing pattern of the hit antennas), the events are rejected as 'repetitive' and unlikely to arise from processes such as neutrinos interacting in-ice.

Application of the above event selection to Testbed data acquired between March 2011 and August 2011 results in one event passing all applied cuts; this event is considered a misidentified in-ice calibration pulser event because its timing and amplitude characteristics are typical of those events.

\section{Results}

No neutrino candidate events were found for the Interferometric Map Analysis and the results from this analysis are used to derive constraints on the neutrino flux. Compared to the Interferometric Map Analysis, the Coherently Summed Waveform Analysis has a 30\% higher analysis efficiency and $\mathrm{a} \sim 10 \%$ lower effective livetime, thus limits derived from the latter give a very similar result.

After finding no neutrino candidate events passing all cuts, we set limits on the neutrino flux given the effective volume of the Testbed derived from AraSim and the total livetime of the period examined. The limit curve shown in Fig. 7 was made for the Interferometric Map Analysis.

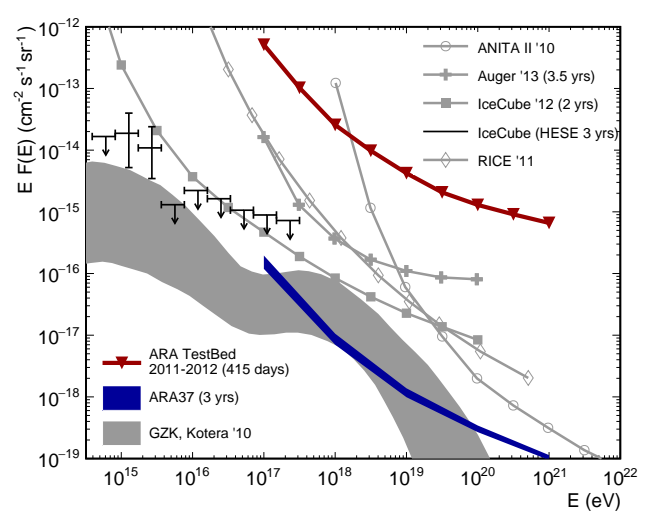

Figure 7: The limits placed compared with the projected ARA37 trigger-level sensitivity and results from other experiments.

\section{References}

[1] Greisen, K., Phys. Rev. Lett., 16, 748 (1966); Zatsepin, G. T. and Kuzmin, V. A., JETP Lett., 4, 78 (1966)

[2] Berezinsky, V. S. and Zatsepin, G. T., Phys. Lett., B28, 423 (1969); Berezinsky, V. S. and Zatsepin, G. T., Sov. J. Nucl. Phys., 11, 111 (1970)

[3] Askaryan, G. A., JETP, 14, 441, (1962); Askaryan, G. A., JETP, 21, 658 (1965); Gorham, Peter et al., Phys. Rev., 62, 8590 (2000); Saltzberg, D. et al., AIP Conf. Proc., 579, 225 (2001); Gorham, Peter W. et al., Phys. Rev., D72, 023002 (2005); Gorham, P. W. et al., Phys. Rev. Lett., 99, 171101 (2007)

[4] Gorham, P. W. et al., Phys. Rev., D82, 022004 (2010); Gorham, P. W. et al., Erratum (2010)

[5] Aartsen, M.G. et al., Phys.Rev., D88, 112008, (2013); Aartsen, M.G. et al., Science, 6161, 342, 1242856 (2013)

[6] Allison, P. et al., Astropart.Phys., 35, 457 (2012)

[7] Barwick, S. and Besson, D. and Gorham, P. and Saltzberg, D., J. Glaciol., 51, 231 (2005)

[8] P. Allison et al., Astroparticle Physics, 70, 0, 62 (2015)

[9] Kotera, K., Allard, D. and Olinto, A. V., JCAP, 1010, 013 (2010) 